\title{
Impacto da depressão e outros fatores psicossociais no prognóstico de pacientes renais crônicos
}

\author{
Paulo Roberto Zimmermann* \\ Juliana Oliveira de Carvalho** \\ Jair de Jesus Mari ${ }^{* *}$
}

\section{INTRODUÇÃO}

"As primeiras três décadas da diálise foram para aumentar a sobrevivência dos pacientes. O desafio para os próximos 30 anos será entender a associação entre fatores psicossociais e evolução dos pacientes, o que inclui ajustamento, adesão, morbidade e mortalidade"1. "Uma quantidade imensa já foi apreendida sobre as reações fisiológicas nos pacientes de diálise. O campo do estresse tem somente iniciado a fazer conexões plausíveis entre emoções e seus mediadores biológicos"2. As duas afirmações acima, escritas por um pesquisador reconhecido nessa área, mostranos claramente a importância do tema ora proposto.

Nos períodos iniciais do uso da diálise,

Trabalho realizado no Hospital São Lucas da Pontifícia Universidade Católica do Rio Grande do Sul.

* Professor Adjunto do Departamento de Psiquiatria e Medicina Legal da PUCRS, Porto Alegre, RS.

** Acadêmica de Medicina da PUCRS, Porto Alegre, RS.

*** Professor Titular do Departamento de Psiquiatria da Universidade Federal de São Paulo, SP. todos os esforços eram dedicados para a manutenção da vida; desde então, progressos muito significativos foram feitos nesse sentido, e, atualmente, essa manutenção já é conseguida de forma mais consistente. Abrese, assim, espaço para outro tipo de preocupação, qual seja a dos aspectos emocionais desses pacientes e a preocupação com sua qualidade de vida. Os trabalhos apresentados até agora em sua maioria são trabalhos de associação entre diversos fatores psicossociais e morbidade e mortalidade. Recentemente começaram a surgir trabalhos que lançam algumas idéias sobre mediadores biológicos, explicando essas associações ${ }^{3}$. Os fatores que mais freqüentemente podem influenciar a evolução dos pacientes renais crônicos podem ser divididos em dois grupos: fixos - aqueles que não podem ser modificados pelo paciente ou médico e incluem idade, doença concomitante, gênero e raça; variáveis - os que podem ser modificados e incluem depressão, suporte social e status marital ${ }^{1}$.

Os autores realizaram uma pesquisa no PubMed, utilizando os termos "hemodialysis", "CAPD", "renal transplantation", "kidney 
transplantation", "depression", "quality of life" e "psychosocial factors". Foram privilegiados os artigos de coorte, uma vez que estes mais se aplicavam à presente pesquisa.

\section{FATORES}

\section{Depressão}

Os achados relativos a depressão nesse grupo de pacientes ainda são muito contraditórios. Isso pode se dever a uma série de fatores, como populações muito diversas, equipes médicas com formação e experiência diferentes, critérios para diagnóstico da depressão não-homogêneos, instrumentos de medida diferentes, entre outros fatores. Um exemplo disso se encontra no trabalho de revisão de Kimmel et al. ${ }^{4}$, que afirmam que a prevalência da depressão nesses pacientes varia de 0 até $100 \%$. Em outro trabalho, Kimmel ${ }^{2}$ afirma que o risco de um quadro de depressão maior durante a vida é de 10 a $25 \%$ em mulheres e de 5 a $12 \%$ em homens. Neste mesmo estudo, o autor afirma que a depressão tende a diminuir com o aumento da idade, mas destaca que este achado deve ser tomado com um certo cuidado, pela possibilidade de um viés do sobrevivente, pois, na medida em que os mais deprimidos morrem, sobrevivem aqueles com menor ou sem depressão. Deoreo ${ }^{5}$, ao examinar um grupo de 1.000 pacientes durante 2 anos, encontrou uma prevalência de depressão de $25 \%$ utilizando como instrumento o SF36, e Lopes et al. ${ }^{6}$ avaliaram 5.256 pacientes em diversos centros dos Estados Unidos e Europa, encontrando uma prevalência de depressão de $20 \%$. Em extenso trabalho que examinou dados de 176.368 pacientes dos registros do Medicare americano, viu-se que $9 \%$ dos pacientes em hemodiálise apresentavam relato de doença mental em seus registros, e que, destes, $26 \%$ consistiam em depressão e transtornos afetivos ${ }^{4}$.

A etiologia da depressão é usualmente associada com alguma perda - e as perdas são normalmente numerosas e duradouras para o paciente com doença renal. Existe a perda da função renal, da sensação de bem-estar, de seu papel tanto na família quanto no trabalho, perda de tempo, de fontes de recursos financeiros, da função sexual, entre outras. A isso se devem acrescentar características de personalidade do paciente, além de uma eventual predisposição genética para a depressão ${ }^{1}$.

Os trabalhos que examinam uma eventual associação entre depressão e morbimortalidade apresentam resultados muitas vezes contraditórios, como veremos a seguir. Alguns estudos de coorte mostraram esta associação: Devins et al. ${ }^{7}$ examinaram 97 pacientes utilizando o Beck Depression Inventory (BDI) num estudo de 46 meses e encontraram associação entre a depressão e a mortalidade, fato também constatado por Wai et al. ${ }^{8}$, que acompanharam 285 pacientes por 18 meses. Outros estudos mostram que não existe uma associação entre depressão e mortalidade: Kimmel et al. ${ }^{9}$, estudando 295 pacientes durante 26 meses e fazendo medidas através do BDI, não encontraram associação, assim como Christensen et al. ${ }^{10}$, que examinaram 84 pacientes durante 3,5 anos com diversas medidas utilizando o BDI, também não encontraram associação, mesmo tendo dividido - BDI entre sua parte cognitiva e a somática.

Lopes et al. ${ }^{6}$, já citados anteriormente, constataram que os pacientes deprimidos apresentavam um risco relativo de morte de 1,23 e, além disso, apresentavam um risco relativo de internações hospitalares de 1,11 quando comparados com pacientes nãodeprimidos. Como o grupo de pacientes era muito grande (5.256) todos os resultados foram significativos do ponto de vista estatístico. Os dados foram corrigidos para o país de origem. Avaliando 174 pacientes em estudo de coorte de 1 ano, Kimmel et al. ${ }^{3}$ não encontraram associação entre depressão e mortalidade. Nesse trabalho, os autores avaliaram a influência da relação diádica do gênero e da depressão na evolução desses pacientes. É um trabalho que começa a investigar a relação entre emoções e mediadores biológicos na medida em que identifica que mulheres em hemodiálise e com boa relação diádica apresentam um nível elevado de betaendorfina, diferentemente do que acontece com homens. Nesse grupo, o nível de interleucina-1 não foi associado a nenhum dos fatores medidos.

Em outro estudo de coorte, os mesmos autores ${ }^{11}$ afirmam que os efeitos da depressão são da mesma magnitude que fatores de risco médico nesses pacientes. Estudando 295 pacientes provenientes de três clínicas diferentes ao longo de 2 anos, com medidas de depressão semestral pelo BDI, constataram que a depressão no início da pesquisa não estava associada a mortalidade, mas que a permanência da depressão trazia um risco relativo de morte de 1,24 (já corrigido para idade, albumina, tipo de dialisador e local da 
diálise). Após ajuste para o tempo, um BDI elevado está associado a um risco relativo de 1,32 , e, mesmo se utilizarmos seu componente cognitivo individualmente (CDI), ele estará associado a um risco relativo de 1,23.

O mesmo trabalho afirma que, estratificando os resultados do BDI, aqueles pacientes classificados como apresentando depressão leve vivem mais do que aqueles classificados como moderados ou graves. Cita também que qualquer programa de atendimento a esses pacientes que possa baixar 8,1 pontos na média do BDI poderá aumentar sua sobrevida em 32\%.

Examinando pacientes em tratamento com diálise peritoneal ambulatorial contínua (CAPD), Finkelstein et al. ${ }^{12}$ constataram que depressão e ansiedade aumentada estavam relacionadas com uma incidência maior de peritonite nesses pacientes, ou seja, com uma morbidade maior. Ao estudar 88 pacientes em situação de transplante renal, Akman et al. ${ }^{13}$ constataram que os pacientes transplantados tinham uma prevalência de depressão menor que os pacientes em lista de espera, e estes tinham índices menores do que aqueles pacientes que perderam o enxerto. Esses autores relataram também que a depressão está inversamente relacionada com o tempo de sobrevida do enxerto, mas que esta afirmação deve ser tomada com certo cuidado, pois existe a possibilidade da depressão ser conseqüência da perda do enxerto, e não sua causa.

Em trabalhos produzidos no Brasil, numa revisão, Almeida \& Meleiro ${ }^{14}$ citam uma prevalência de depressão de 5 a 25\% quando utilizados critérios mais estritos de diagnóstico. A tentar explicar as conseqüências da depressão nesses pacientes, os autores citam que ela tem impacto importante na qualidade de vida, taxas de suicídio, aderência aos tratamentos e mortalidade.

Quanto a isso, outros autore ${ }^{15}$ afirmam que a depressão provoca diminuição da imunidade, relaxamento dos cuidados pessoais, menor adesão a tratamentos e dietas, intensificação dos problemas tanto financeiros e profissionais quanto familiares e, por fim, maior possibilidade de comorbidade com abuso ou dependência de álcool ou drogas.

Em trabalho realizado em Porto Alegre com 41 pacientes em hemodiálise, Duarte et al. ${ }^{16}$ encontraram uma prevalência de depressão maior de $24,39 \%$ de transtorno depressivo maior em remissão de $12,9 \%$, transtorno depressivo menor em remissão parcial de $9,76 \%$ e, por fim, $12,9 \%$ de distimia.

\section{ldade}

Todos os trabalhos revisados mostram que existe uma associação direta entre idade e mortalidade ${ }^{5,7-10,17}$. Os fatores relacionados a idade que podem explicar tais achados não são difíceis de identificar: na medida em que esta aumenta, uma série de fatos ocorre concomitantemente: maior possibilidade de comorbidades, maior incidência de depressão, perdas diversas (seja de cônjuges, suporte social ou capacidade econômica), o próprio processo de envelhecimento, entre outros. Desta forma, a afirmação de que o aumento da idade está associado ao aumento da mortalidade deve ser visto com cuidado, uma vez que esta associação pode se dever à soma de todos esses fatores, e não especificamente à idade. Talvez devêssemos, no futuro, desenvolver pesquisas corrigindo esses fatores. Em estudo de coorte, Devins et al. ${ }^{7}$ avaliaram 97 pacientes em hemodiálise, diálise peritoneal ambulatorial contínua e transplantados durante 4 anos. Os autores constataram que a idade e o número de comorbidades estavam inversamente associados à sobrevida dos pacientes.

Kimmel et al. ${ }^{9}$ estudaram 295 pacientes durante 26 meses de evolução, com avaliações semestrais, e puderam ver que os únicos fatores vinculados à mortalidade eram idade e gravidade da doença. Em trabalho antigo, publicado em 1981 por Wai et al. ${ }^{8}$, foram seguidos 285 pacientes em 14 hospitais diferentes por 18 meses. Os autores relatam que os fatores psicológicos parecem não só interferir no ajustamento desses pacientes, mas também contribuir para comportamentos autodestrutivos. Constataram também uma associação entre idade e mortalidade. Em estudo de revisão, Valderrabano et al. ${ }^{17}$ encontraram trabalhos que relatam o mesmo fato da associação entre idade e mortalidade. Além disso, também descreveram que o aumento da idade está associado à diminuição da qualidade de vida dos pacientes, em especial nos aspectos físicos. Ainda, observaram que é possível que, nos pacientes mais idosos, o impacto de uma doença renal terminal seja menor do que nos jovens, uma vez que eles têm maior capacidade de lidar com as limitações que a doença impõe. O impacto, por exemplo, de três sessões semanais de hemodiálise numa pessoa aposentada é muito menor que numa pessoa em atividade produtiva.

Em estudo de coorte, Christensen et al. ${ }^{10}$ examinaram 84 pacientes durante 3,5 anos e constataram que a idade estava relacionada 
com a mortalidade observada neste período de tempo. Um interessante trabalho publicado por Kutner et al. ${ }^{18}$ relata o seguimento de um grupo de 287 pacientes velhos (acima de 60 anos) por 12 anos. O estudo constatou que, nessa faixa etária, o fator idade não estava associado a mortalidade. Deoreo ${ }^{5}$ conduziu um estudo durante 2 anos com um grupo de 1.000 pacientes e descreveu uma associação positiva entre idade, número de hospitalizações e mortalidade. Além disso, constatou também que os pacientes mais jovens mais freqüentemente faltavam a uma ou mais sessões de hemodiálise por mês.

\section{Gênero}

A maioria dos trabalhos que estuda a possível associação entre gênero e mortalidade aponta no sentido de que não existe associação entre ambos. Os mesmos autores citados anteriormente, Devins et al. ${ }^{7}$, assim como Deoreo $^{5}$, afirmam que essa associação não existe. Valderrabano et al. ${ }^{17}$ relatam estudos que demonstram que a mulher apresenta piores índices de qualidade de vida (morbidade) do que o homem, sugerindo que isso possa acontecer pela perda de seu papel social e pela maior incidência de depressão neste grupo. Leggat et al. ${ }^{19}$, quando estudaram adesão a tratamento, o que influencia a evolução desses pacientes, constatou que, no grupo de 6.251 examinados, o gênero não influenciava a adesão dos pacientes; isso nos permite sugerir, por conseqüência, que não influenciava sua mortalidade. Associação entre gênero e mortalidade foi encontrada no trabalho de Kutner et al. ${ }^{18}$, que examinaram 287 pacientes com mais de 60 anos e encontraram que os homens morreram mais que as mulheres num período de acompanhamento de 2 anos. Talvez este resultado expresse a realidade de que as mulheres vivem mais do que os homens, e não especificamente algum fato relacionado a gênero e pacientes renais.

\section{Raça}

A eventual associação da raça com morbidade e mortalidade é bastante complexa. Perneger et al. ${ }^{20}$, em interessante estudo, citam que as diferenças de estado de saúde nas diferentes raças podem ser causadas, pelo menos em parte, por questões de status socioeconômico. Seguem os mesmos autores afirmando que uma raça minoritária pode ter características genéticas que a predisponham para alguma doença ou algum desfecho. Além disso, raças minoritárias com freqüência apresentam um status socioeconômico menor, o que pode fazer com que os empregos obtidos por seus membros sejam de nível inferior, expondo a pessoa a riscos maiores. Além disso, dificuldades socioeconômicas também podem levar a limitações no acesso à saúde, o que diminui a prevenção de doenças ou seu controle, uma vez instaladas. Apesar disso, na pesquisa que o mesmo autor conduziu com 716 pacientes e 361 controles em Maryland, constatou-se que a raça negra apresenta uma odds ratio de 5,5 para mortalidade, mesmo após a correção para variáveis socioeconômicas.

Leggat et al. ${ }^{19}$, examinando um grupo de 6.251 pacientes, perceberam que os pacientes negros têm uma possibilidade maior de faltar a sessões de hemodiálise ou de interrompê-las antes quando comparados aos pacientes brancos. Contraditoriamente, os autores citam que esses pacientes têm uma chance menor de morte na hemodiálise que os pacientes brancos. Valderrabano et al. ${ }^{17}$ citam estudos que relatam que os pacientes negros em hemodiálise evoluem melhor do que os brancos. Além disso, citando um trabalho de Welch \& Austin, afirmam que a qualidade de vida dos pacientes negros submetidos à hemodiálise é semelhante à dos pacientes brancos e que os autores entendem que raça não necessitaria ser monitorizada nesses pacientes. No mesmo sentido, vários trabalhos ${ }^{7,10,11,13,18,21-23}$, quando descrevem as características demográficas dos grupos estudados, nem citam a raça como um parâmetro.

\section{Suporte social}

Uma definição para suporte social poderia ser a de que o indivíduo percebe ser parte de uma rede de afeto, mútua ajuda e obrigações ${ }^{1}$. Kimmel, em outro trabalho ${ }^{2}$, coloca que o suporte social percebido e recebido de amigos está relacionado ao comparecimento a sessões de hemodiálise, ou seja, à adesão ao tratamento. Além disso, ao relatar o suporte social que um paciente recebe de seus familiares, o autor destaca que isso contribui positivamente para a sua evolução, mas que o paciente é uma fonte muito grande de estresse para a família. O autor cita que as diferenças de suporte social podem estar implicadas nas diferenças de taxas de mortalidade entre países, grupos ou unidades de tratamento e possivelmente contribuem para diferentes taxas de adesão a tratamentos. 
Shidler et al. ${ }^{24}$, estudando 50 pacientes com insuficiência renal crônica (clearance da creatinina menor que 80 ), lançaram a idéia de que o suporte social tem uma ação semelhante a um buffer, ou seja, não apresenta uma ação protetora maior para situações de estresse leves, mas previne ou reduz os efeitos negativos em situações de estresse elevado. Em trabalho já citado, Kimmel et al. ${ }^{3}$, ao estudar 174 pacientes em hemodiálise, encontraram que o suporte social está vinculado ao aumento da sobrevida nesses pacientes.

\section{Status marital/suporte familiar}

A abordagem desta variável está profundamente relacionada com a anterior, uma vez que a família ou a relação de casal na maior parte das vezes está incluída na rede de suporte desses pacientes. Examinando 84 pacientes recebendo hemodiálise em estudo de coorte de 3,5 anos, Christensen et al. ${ }^{10}$, utilizando a Family Environment Scale (FES), que mede coesão, expressividade e conflito, encontraram que o suporte familiar está relacionado à sobrevida dos pacientes. Destacam que o aumento de 1 ponto no resultado dessa escala contribui para uma redução de $13 \%$ na mortalidade deste grupo. Aqueles pacientes situados em um desvio padrão acima da média foram chamados de high family support, e aqueles com um desvio abaixo da média, de low family support. Os autores constataram que, ao longo de 5 anos, a diferença na mortalidade era de três vezes: $18 \%$ dos pacientes high support haviam morrido, contra $52 \%$ dos low support. Além disso, os autores citam que essas diferenças não foram causadas por problemas de adesão, uma vez que os valores de uréia e creatinina não eram diferentes entre os grupos.

Steidl et al. ${ }^{25}$, examinando as famílias de 33 pacientes em diálise, constataram que um funcionamento familiar que contemple coalizão parental, respeito a individualidade em contexto de intimidade e interações calorosas, afetivas e otimistas está relacionado às condições médicas. Além disso, os autores citam que as limitações impostas pela doença também influenciam a família. Em estudo de pacientes transplantados renais, Akman et al. ${ }^{13}$ afirmam que uma porcentagem menor de depressão foi observada entre os pacientes casados, sugerindo que eles se adaptaram mais rapidamente à situação, com um melhor suporte.

Devins et al. ${ }^{7}$, ao estudar, durante 46 hemodiálise, CAPD e transplantados, não encontraram associação entre status marital e mortalidade. Ampliando um pouco mais a influência da doença na família, $\mathrm{Kimmel}^{2}$ cita que existem trocas de papéis na família com seu aparecimento e que, além disso, as alterações sexuais freqüentes também geram sentimentos diversos, que devem ser manejados. O estudo também relata que, se existir uma relação marital boa, haverá um aumento na saúde dos pacientes. O mesmo autor, estudando as relações diádicas desses pacientes, constatou que as mulheres que tinham valores de um desvio padrão da média na Dyadic Adjustment Scale apresentavam uma redução de $50 \%$ no risco de mortalidade e que uma diminuição no escore negatividade da mesma escala estava associada a uma redução de $60 \%$ no risco. Esses achados não se confirmaram com os pacientes homens.

\section{CONCLUSÕES}

Como ficou demonstrado durante todo o trabalho, não existem resultados conclusivos sobre a influência dos fatores emocionais na morbidade e mortalidade desses pacientes, embora algumas afirmações possam ser feitas.

A influência da depressão na evolução dos pacientes é o fator mais estudado dentre todos. Os resultados ainda não são conclusivos, mas existe uma tendência à associação direta entre depressão, morbidade e mortalidade nesse grupo de pacientes. Devido à possibilidade de tratamento desses quadros, evitando possíveis conseqüências graves na evolução desses pacientes, os autores acham importante que mais estudos sejam realizados nessa área, o que permitiria que se atingisse um grau de certeza maior quanto à verdadeira influência da depressão em pacientes renais crônicos.

Existe uma concordância em todos os trabalhos revisados, exceto $\mathrm{um}^{18}$, de que a idade está relacionada diretamente com a mortalidade. Os trabalhos, contudo, não corrigem os resultados obtidos quanto a outras variáveis mais incidentes nesse grupo de pacientes, como o aumento de comorbidades, o aumento da depressão, a diminuição de suporte social por perda de esposos, etc. Ou seja, não está claro se é a idade individualmente a responsável pelo aumento da mortalidade ou algum outro fator vinculado a ela.

Quanto ao gênero, os trabalhos indicam uma forte tendência no sentido de não existir uma associação com morbidade ou mortalidade. 
Quanto à raça, os resultados são contraditórios, sendo que a tendência é de que ela não influencie a morbidade ou mortalidade desses pacientes. Além disso, os resultados eventualmente positivos podem ser motivados por outros fatores vinculados às raças dos pacientes. Muitos dos trabalhos revisados nem descrevem a raça dos pacientes entre as características demográficas.

O suporte social e familiar parece estar inversamente associado com a morbidade ou mortalidade, e, mais uma vez, outros fatores amplamente envolvidos no suporte social podem ser os responsáveis pelos resultados.

Como não se trata de uma pesquisa do tipo meta-análise, os resultados devem ser vistos com algum cuidado, uma vez que não são uniformes, devido a diferentes populações, critérios diagnósticos, tempo de observação, etc.

Por fim, existe a necessidade de estudos longitudinais, melhor controlados, com metodologias bem definidas, grupos de pacientes maiores e observados por mais tempo para que se possa chegar a resultados mais válidos.

\section{REFERÊNCIAS BIBLIOGRÁFICAS}

1. Kimmel PL. Psychosocial factors in adult end-stage renal disease patients treated with hemodialysis: correlates and outcomes. Am J Kidney Dis 2000;35(4):S132-40.

2. Kimmel PL. Psychosocial factors in dialysis patients. Kidney Int 2001;59:1599-613.

3. Kimmel PL, Peterson RA, Weihs KL, Shidler N, Simmens SJ, Alleyne S, et al. Dyadic relationship conflict, gender, and mortality in urban hemodialysis patients. J Am Soc Nephrol 2000;11:1518-25.

4. Kimmel PL, Thamer M, Richard CM, Ray NF. Psychiatric illness in patients with end-stage renal disease. Am J Med 1998;105:214-21.

5. Deoreo PB. Hemodialysis patients - assessed functional health status predicts continued survival, hospitalization, and dialysis-attendance compliance. Am J Kidney Dis 1997;30(2):204-12

6. Lopes AA, Bragg J, Young E, Goodkin D, Mapes D, Combe $\mathrm{C}$, et al. Depression as a predictor of mortality and hospitalization among hemodialysis patients in the United States and Europe. Kidney Int 2002;62:199-207.

7. Devins GM, Mann J, Mandin H, Paul LC, Hons RB, Burgess ED, et al. Psychosocial predictors of survival in end-stage renal disease. J Nerv Ment Dis 1990;178(2):127-33.

8. Wai L, Richmond J, Burton H, Lindsay RM. Influence of psychosocial factors on survival of hemo-dialysis patients. Lancet 1981;2(8256):1155-6.

9. Kimmel PL, Peterson RA, Weihs KL, Simmens SJ, Alleyne S, Cruz I, et al. Psychosocial factors, behaviora compliance and survival in urban hemodialysis patients. Kidney Int 1998;54:245-54

10. Christensen AJ, Wiebe JS, Smith TW, Turner CW. Predictors of survival among hemodialysis patients: effect of perceived family support. Health Psychol $1994 ; 13(6): 521-5$.
11. Kimmel PL, Peterson RA, Weihs $\mathrm{KL}$, Simmens SJ, Alleyne S, Cruz I, et al. Multiple measurements of depression predict mortality in a longitudinal study of chronic hemodialysis outpatients. Kidney Int 2000;57:2093-8.

12. Finkestein FO, Finkestein $\mathrm{SH}$. Depression in chronic dialysis patients: assessment and treatment. Nephrol Dial Transplant 2000;15:1911-3.

13. Akman B, Ozdemir FN, Sezer S, Micozkadioglu H, Haberal M. Depression levels before and after renal transplantation. Transplant Proc 2004;36:111-3.

14. Almeida AM, Meleiro, AMAS. Revisão: Depressão e insuficiência renal crônica: uma revisão. J Bras Nefrol 2000;22(1):192-200.

15. Levenson JL, Glochesky S. Psychological factors affecting end-stage renal disease. A review. Psychosomatics 1991;32(4):382-9

16. Duarte AP, Mattevi BS, Berlim MT, Morsch C, Thomé FS, Barros EJ, et al. Prevalência da depressão maior nos pacientes em hemodiálise crônica. Rev HCPA 2000;20(3):240-6.

17. Valderrabano F, Jofre R, Lopez-Gomez JM. Quality of life in end-stage renal disease patients. Am J Kidney Dis 2001;38(3):443-64.

18. Kutner NG, Lin LS, Fielding B, Brogan D, Hall WD. Continued survival of older hemodialysis patients: investigation of psychosocial predictors. Am J Kidney Dis $1994 ; 24(1): 42-9$

19. Leggat JE Jr, Orzol SM, Hulbert-Shearon TE, Golper TA, Jones CA, Held PJ, et al. Noncompliance in hemodialysis: predictors and survival analysis. Am J Kidney Dis 1998;32(1):139-45.

20. Perneger TV, Whelton PK, Klag MJ. Race and end-stage renal disease. Socioeconomic status and access to health care as mediating factors. Arch Intern Med 1995; $155: 1201-8$

21. Merkus MP, Jager KJ, Dekker FW, de Haan RJ, Boeschoten EW, Krediet RT. Predictors of poor outcome in chronic dialysis patients: the Netherlands cooperative study on the adequacy of dialysis. Am J Kidney Dis 2000;35(1):69-79.

22. Gorlen T, Ekeberg O, Abdelnoor M, Enger E, Aarseth HP. Quality of life after kidney transplantation. A $10-22$ years follow-up. Scand J Urol Nephrol 1993;27:89-92.

23. Pisani F, Vennarecci G, Tisone G, Buonomo O, laria G, Rossi S, et al. Quality of life and long-term follow-up after kidney transplantation: a 30-year clinical study. Transplant Proc 1997;29:2812-3

24. Shidler NR, Peterson RA, Kimmel PL. Quality of life and psychosocial relationship in patients with chronic renal insufficiency. Am J Kidney Dis 1998;32(4):557-66.

25. Steidl JH, Finkelstein OF, Wexler JP, Feigenbaum $H$, Kitsen J, Kliger AS, et al. Medical condition, adherence to treatment regimens, and family functioning. Arch Gen Psychiatry 1980;37:1025-7.

\section{RESUMO}

Os autores realizaram uma revisão bibliográfica sobre a depressão e outros fatores psicossociais que poderiam influenciar a evolução dos pacientes renais. Avaliaram trabalhos relativos a depressão, idade, gênero, raça, suporte social e status maritall suporte familiar. Ao final, ficou demonstrado que não existem resultados definitivos nas pesquisas realizadas sobre esses fatores. $A$ idade parece ser um fator que influencia a evolução, mas os fatores de confusão não foram contemplados nos trabalhos 
examinados, e surgiram evidências fortes de que o gênero não estaria associado à evolução desses pacientes.

Descritores: Depressão, fatores psicossociais, nefrologia, diálise renal, transplante de rim, diálise peritoneal ambulatorial contínua.

\section{ABSTRACT}

The authors review the literature on depression and other psychosocial risk factors that may influence the evolution of patients with kidney disease. Studies addressing depression, age, gender, skin color, social support, and marital status/family support were included in the review. The study revealed that trials assessing these factors have not come to a consensus so far. Age seems to be a factor that interferes with disease evolution, but the papers examined did not include confounding factors. Some papers also showed strong evidence that gender is not associated with the evolution of these patients.

Keywords: Depression, psychosocial factors, nephrology, renal dialysis, kidney transplantation, peritoneal dialysis, CAPD.

Title: The impact of depression and others psychosocial factors in the prognosis of chronic renal patients

\section{RESUMEN}

Los autores realizaron una revisión bibliográfica sobre la depresión y otros factores psicosociales que podrían tener influencia en la evolución de los pacientes renales. Evaluaron trabajos relativos a depresión, edad, género, raza, soporte social y situación marital / soporte familiar. Al final, se demostró que no hay resultados definitivos en las investigaciones realizadas sobre estos factores. La edad parece ser un factor que influye en esta evolución, pero los factores de confusión no se contemplaron en los trabajos examinados. Además, aparecieron fuertes evidencias de que el género no estaría asociado a la evolución de estos pacientes.

Palabras clave: Depresión, factores psicosociales, nefrología, diálisis renal, trasplante de riñón, diálisis peritoneal ambulatoria continua.

Título: El impacto de la depresión y otros factores psicosociales en el pronóstico de pacientes renales crónicos

Correspondência:

Paulo Roberto Zimmermann

Rua Carvalho Monteiro, 257/301 - Bela Vista

CEP 90470-100 - Porto Alegre - RS

E-mail: przimmer@terra.com.br

Copyright (C) Revista de Psiquiatria

do Rio Grande do Sul - SPRS 